\title{
Change in Seroprevalence of Total Antibodies to COVID-19 Virus among Healthcare Workers in a Tertiary Care Hospital, Mumbai
}

Sweta Shah ${ }^{1}$, Pooja Thakkar ${ }^{2}$, Tanu Singhal ${ }^{3}$, Aamreen Kazi ${ }^{4}$, Reshma Tejam ${ }^{5}$

\begin{abstract}
Serological tests for estimation of total antibodies to SARS-CoV-2 for healthcare workers (HCWs) help to understand the epidemiology of COVID-19 disease in the healthcare setting.

Aims and objective: To note the seroprevalence of COVID-19 among HCWs in a tertiary care hospital.

Settings and design: This study was done at a tertiary care hospital, Mumbai. Detection of the "Total antibody to SARS-CoV-2" virus was offered as part of the health check-up from September 24, 2020, to December 31, 2020, after taking due consent and history of the HCWs.

Materials and methods: The test was anti-SARS-CoV-2 on Elecsys, Roche Diagnostics by ECLIA.

Results: Five hundred and seventy (25.8\%) HCWs out of a total of 2,211 working in the hospital were tested for total antibodies against the SARS-CoV-2 virus. Two hundred and forty-eight (43.5\%) HCWs were positive for the total antibody test to SARS-CoV-2 virus. $86.8 \%$ of the HCWs who were diagnosed to have COVID-19 in past were seropositive. $65.2 \%$ of HCWs even when were PCR negative but had been in contact with an infected person but were PCR negative developed antibodies. Twenty-eight percent of HCWs did not have any symptoms of COVID-19 infection or were not in close contact of positive contact were seropositive. Of these 248 antibody-positive HCWs, 105 (42.3\%) had tested positive for RT-PCR in the past, 30 (12.1\%) were symptomatic/close contacts of COVID-19 patients but had tested negative for RT-PCR in the past and 113 (45.6\%) were not symptomatic, had no high-risk contact and were never tested.

Conclusion: There is a marked increase in seroprevalence rates in asymptomatic HCWs from 4.3\% in June 2020 to $28 \%$ between September and December 2020 at our center.

Clinical significance: An increase in COVID-19 seroprevalence among HCWs indicates continued subclinical exposure during and after the first wave during a global pandemic and this should be used to predict the next surge of infections.

Keywords: COVID-19, Healthcare workers, SARS-CoV-2, Seroprevalence, Total antibodies.

Indian Journal of Medical Biochemistry (2021): 10.5005/jp-journals-10054-0170
\end{abstract}

\section{INTRODUCTION}

The risk of front-line healthcare workers (HCWs) acquiring COVID-19 is higher than that of the general community. Among HCWs, the risk is highest for those working in inpatient settings (24.30\%) and nursing homes (16.24\%). ${ }^{1}$ Therefore, prevention, early diagnosis, isolation, and treatment of $\mathrm{HCW}$ are crucial to maintaining continuity of patient care and prevention of onward transmission to patients. The study site has been treating COVID-19 patients since March 2020 along with non-COVID-19 patients and follows protocols for infection control and diagnosis, treatment, isolation of infected HCWs in accordance with national guidelines. ${ }^{2}$

Serological tests for estimation of COVID-19 antibodies in HCWs are recommended by the Indian Council of Medical Research (ICMR) and other agencies to understand the epidemiology of the disease and the effectiveness of infection control measures. ${ }^{3,4}$ The seroprevalence rates in the last quarter of the year changed significantly than those were reported in a small cohort of HCWs in June $2020 .{ }^{5}$ This study reveals high seroprevalence among HCWs at the end of the first wave of the COVID-19 epidemic in our institute.

\section{Materials and Methods}

This study was done at a tertiary care hospital, Mumbai, India. All HCWs with any COVID-19-related symptoms or asymptomatic HCW if they had close/household contact with COVID-19 patients \begin{tabular}{l}
\hline 1,2,4Department of Microbiology, Kokilaben Dhirubhai Ambani \\
Hospital and Research Institute, Mumbai, Maharashtra, India \\
${ }^{3}$ Department of Infectious Diseases, Kokilaben Dhirubhai Ambani \\
Hospital and Research Institute, Mumbai, Maharashtra, India \\
${ }^{5}$ Infection Prevention and Control, Kokilaben Dhirubhai Ambani \\
Hospital and Research Institute, Mumbai, Maharashtra, India \\
Corresponding Author: Sweta Shah, Department of Microbiology, \\
Kokilaben Dhirubhai Ambani Hospital and Research Institute, Mumbai, \\
Maharashtra, India, Phone: +91 932025589, e-mail: sweta.shah@ \\
kokilabenhospitals.com \\
How to cite this article: Shah S, Thakkar P, Singhal T, et al. Change \\
in Seroprevalence of Total Antibodies to COVID-19 Virus among \\
Healthcare Workers in a Tertiary Care Hospital, Mumbai. Indian J Med \\
Biochem 2021;25(1):42-45. \\
Source of support: Nil \\
Conflict of interest: None
\end{tabular}

were tested by SARS-CoV-2 RT-PCR since March 2020 as part of the hospital. The HCWs of the hospital undergo free health check-ups annually. Detection of the "Total antibody to SARS-CoV-2" virus was offered as part of the health check-up from September 24, 2020, onward. After taking informed consent from the HCW, details of the past history of COVID-19-related illness and SARS-CoV-2 RT-PCR 
results and antibody results in the past were recorded. All HCWs who were scheduled for health checks were offered the test irrespective of their previous SARS-CoV-2 RT-PCR report. Total antibody detection test "Anti-SARS-CoV-2", on Elecsys, Roche Diagnostics by ECLIA which uses a recombinant protein representing the nucleocapsid $(N)$ Antigen for detection. All HCWs were provided with the test results and counseled. RT-PCR was done by commercial ICMR approved kits on open platform thermal cycler Rotorgene, Qiagen. The data then were anonymized and further calculated for epidemiological research.

\section{Results}

Five hundred and seventy (25.8\%) HCWs out of a total of 2,211 working in the hospital were tested for total antibodies against the SARS-CoV-2 virus from September 24, 2020, to December 31, 2020. These included doctors, nurses, therapists, technicians, and nonclinical hospital office staff. Thus, the group tested was a random mix of HCWs working in a high-risk area and administrative area. However, it did not include the housekeeping staff of the hospital. The age group of the staff is between 22 years and 60 years. Two hundred and forty-eight (43.5\%) HCWs were positive for the total antibody test to SARS-CoV-2 virus. The monthly seropositivity over the study duration was similar and there was no decrease in the seropositivity from September to December 2020 as mentioned in Table 1.

$86.8 \%$ of the HCWs who were diagnosed to have COVID-19 in past were seropositive. All the HCWs had mild to moderate COVID-19 disease. None of them needed ICU care. $65.2 \%$ of HCWs even when were RT-PCR negative but had been in contact with an infected person but were RT-PCR negative developed antibodies. Twenty-eight percent of HCWs did not have any symptoms of COVID-19 infection or were not in close contact of positive contact were seropositive. Thus, 31.8\% (143 out of $449 \mathrm{HCWs)} \mathrm{developed}$ antibodies when did not have any disease (Table 2).

Of these 248 antibody-positive HCWs, 105 (42.3\%) had tested positive for RT-PCR in the past, 30 (12.1\%) were symptomatic/close contacts of COVID-19 patients but had tested negative for RT-PCR in the past, and 113 (45.6\%) were not symptomatic, had no high-risk contact and were never tested as shown in Table 3.

Sixteen HCWs were SARS-CoV-2 RT-PCR positive but had negative antibody test results. Ten HCWs who were seronegative at the beginning of the study developed RT-PCR confirmed infection during the study period. Of the remaining six, four HCWs had infections 4-6 months before antibody testing while two HCWs had infections 1-3 months before antibody testing. Thus, antibodies among HCWs might have depleted or they did not develop a demonstrable humoral immune response. Furthermore, none of the seropositive HCW developed SARS-CoV-2 infection in the study period.

\section{Discussion}

We report here a marked increase in seroprevalence rates among HCWs from 4.3\% in June 2020 to $43.5 \%$ between September and
December 2020 at our center. The data across various centers in India have shown similar changes in seroprevalence as the first wave of the infection was at its peak from June to August 2020 in India.

It also should be noted that IgG assay and total antibody assays are the qualitative types of assays. The purpose of the assay is to see that an immune response was mounted to COVID-19 infection. These assays are not used for diagnosis of infection and not used as a clinical tool for the course of illness or outcome of the disease but are to be used only for serosurveillance as per ICMR. Hence, the comparison between total antibodies assay and IgG assays is considered valid.

The first national SARS-CoV-2 serosurvey in India, done in May to June 2020, found a SARS-CoV-2 IgG antibody seroprevalence of $0.73 \%$ which has increased to $7.1 \%(95 \% \mathrm{Cl} 6.2-8.2)$ between August and September 2020. ${ }^{6}$ National Survey study indicated that nearly 1 in 15 individuals aged 10 years or older were exposed to SARS-CoV-2 in India by August 18, 2020, although significant number remained either asymptomatic or untested. ${ }^{6}$ Study done in Mumbai in June 2020 demonstrated adjusted seroprevalence was higher in slums (means ranging from 55.1 to 61.4\%) than in nonslums (mean ranging from 12.0 to $18.9 \%$ ) across the various area of city. ${ }^{7}$ Another study was done in Devarajeevanahalli, Karnataka in June to July 2020 reported the overall seroprevalence of IgG antibodies as $57.9 \%^{8}$ which declined from $28.39 \%$ (August) to $24.08 \%$ (September), and $24.71 \%$ (October). The overall prevalence of COVID-19 in Karnataka from September 3 to 16, 2020, was 27.3\%, including lgG $16.4 \%$ and active infection $12.7 \%$ where 16,416 people were surveyed. ${ }^{9}$ In July 2020 in Chennai reported seroprevalence of $18.4 \%$ where 12,405 serum samples were tested. Furthermore, an overall positivity of $32.3 \%$ was reported in October 2020 in Chennai (2,062 were positive for the presence of Ig G antibodies out of 6,389 samples). ${ }^{10}$ Thus, our changing seroprevalence matches with the others in the country.

Table 2: Correlation of result of serological tests and RT-PCR for SARSCoV-2

\begin{tabular}{lllll}
\hline & $\begin{array}{l}\text { Total tests } \\
\text { done }\end{array}$ & $\begin{array}{l}\text { Previous RT- } \\
\text { PCR pos }\end{array}$ & $\begin{array}{l}\text { Previous RT- } \\
\text { PCR neg }\end{array}$ & $\begin{array}{l}\text { RT-PCR not } \\
\text { done }\end{array}$ \\
\hline $\begin{array}{l}\text { Antibody } \\
\text { reactive }\end{array}$ & 570 & 121 & 46 & 403 \\
$\begin{array}{l}\text { Percentage } \\
\text { Pents }\end{array}$ & 43.5 & 105 & 30 & 113 \\
\hline
\end{tabular}

Table 3: Correlation of previously confirmed diagnosis by RT-PCR with seroprevalence

\begin{tabular}{lcl}
\hline & Antibody reactive & Percentage \\
\hline Previous RT-PCR positive & 105 & 42.3 \\
Previous RT-PCR negative & 30 & 12.1 \\
RT-PCR not done & 113 & 45.6 \\
Total antibody reactive & 248 & \\
\hline
\end{tabular}

Table 1: Monthly seroprevalence of HCWs over the study duration

\begin{tabular}{lllccc}
\hline Month & September & October & November & December & Total \\
\hline Total tests done & 41 & 219 & 169 & 141 & 570 \\
$\begin{array}{l}\text { Total specimen those } \\
\text { were reactive }\end{array}$ & 17 & 101 & 65 & 65 & 248 \\
\begin{tabular}{l} 
Percentage \\
\hline
\end{tabular} & 41.5 & 46.1 & 38.5 & 46.1 & 43.5 \\
\hline
\end{tabular}


$86.8 \%$ of the HCWs who were diagnosed to have COVID in past were seropositive. The diagnosis of COVID could have happened any time from March to September 2020 and hence some of the staff may have lost antibodies or they were non-responders for humoral immunity and probably developed the only cell-mediated immunity. $65.2 \%$ of HCWs even when were PCR negative but had been in contact with an infected person but were PCR negative developed antibodies. Twenty-eight percent of HCWs did not have any symptoms of COVID-19 infection or were not in close contact of positive contact were seropositive. This is significantly higher ( $p$ $<0.05$ ) than $4.3 \%$ reported in June 2020 . Thus, 31.8\% (143 out of 449 HCWs) developed antibodies when did not have any disease. The significant increase also may be due to recurrent exposure of staff to the virus both in the community and in the hospital. Apart from HCWs working in a high-risk area, staying in a "metro" city of high density during the peak of the first wave and traveling to work with inadequate social distance every day could have contributed to significantly high seropositivity.

In a study done by Baveja et al., ${ }^{11}$ the seroprevalence among HCWs was reported as 6.9\% (similar between COVID and non-COVID areas) in May 2020 in Mumbai, which is similar to our institute data in June 2020.

Results from a tertiary care hospital, NCR states that out of 3,739 HCWs tested, 487 (13\%) were positive for total SARSCoV-2 antibodies from January to February 2021. The highest seroprevalence was observed in administrative staff (19.6\%) and least in physicians $(5.4 \%){ }^{12}$

Our study may underestimate COVID-19 exposure as individuals with mild disease/asymptomatic infection may not mount an immune response or may have lost antibodies by the time they were tested. In our cohort too, six HCWs were PCR positive but were seronegative.

Ten HCWs who were seronegative developed infection during the study period. But no seropositive HCWs who were previously untested by RT-PCR developed a new infection in the study duration. So, there is possibly, a correlation between measurable total antibodies and immunity against reinfection.

However, with the emergence of immune escape variants like the South African/Brazilian variants, we may start seeing infections in previously immune/infected/vaccinated HCWs. If such infection is documented, the isolates should be subjected to whole-genome sequencing to detect the variants.

The limitations of our study are the small sample size, no risk stratification among the HCWs, and random sampling method.

Nonetheless, a significant increase in seroprevalence among asymptomatic HCWs at the end of the first wave of the COVID-19 pandemic is noteworthy. It has been postulated that there may be a decline in antibody level after 3-6 months duration. However, $25.8 \%$ of our HCWs were positive when tested and $46 \%$ of those had never developed any symptoms. Hence, this probably indicates that the HCWs have often suffered subclinical exposure to the virus when at work or while traveling to work, or while living in a busy community of an overcrowded metro city and have developed and retained antibodies. In a study done in Mumbai, ${ }^{13} 46$ out of $49 \mathrm{HCWs}$ (98\%) were seropositive after 6 months of initial testing and $81 \%$ of HCWs had actually risen in the optical density (OD) while $19 \%$ had a reduction in OD which also demonstrates the same phenomenon.

These high levels of seroprevalence might have contributed to the delayed second wave of infection in India. Larger serosurveys in HCWs and comparing them to the general population will help to understand the epidemiology of the illness. These could be important analytes for making a mathematical model for the prediction of disease epidemiology and expectation of the third wave apart from the vaccination itself.

Vaccination was initiated in the 2nd week of January among HCWs in India followed by the elderly population and was opened for all since the first week of May. However, the availability of vaccines for the vast Indian population will remain a challenge for an initial couple of months. Thus, antibody prevalence after vaccination and their ability to prevent infection by mutants and reinfection will need another wide study.

\section{Conclusion}

An increase in COVID-19 seroprevalence among HCWs from 4.3\% in June to $43.5 \%$ in the study period is a significant rise despite an increase in seroprevalence in the community. It indicates continued subclinical exposure during and after the first wave during the global pandemic in both, workplace and community. These data should be considered to understand the epidemiology, predict the next surge of infections.

\section{Clinical Significance}

An increase in COVID-19 seroprevalence among HCWs indicates continued subclinical exposure during and after the first wave during the global pandemic and this should be used to predict the next surge of infections.

\section{ACKnOWLedgments}

We acknowledge the hospital management for performing total antibodies to SARS-CoV-2 in annual health checks for all the staff.

\section{Manufacturer's Name}

Total Antibodies to SARS-CoV-2: ECLIA on Elecsys, Roche, Switzerland.

\section{References}

1. Nguyen LH, Drew DA, Graham MS, et al. Risk of COVID-19 among front-line health-care workers and the general community: a prospective cohort study. Lancet 2020;5(9):E475-E483. DOI: 10.1016/ S2468-2667(20)30164-X.

2. Government of India, Clinical management of COVID 19. Available from: https://www.mohfw.gov.in/pdf/ UpdatedClinicalManagementProtocolforCOVID19 dated 03072020. pdf. [Last accessed on 2020 Jul 04].

3. Indian Council of Medical Research. Newer Additional Strategies for COVID-19Testing. Available from: https://www.icmr.gov.in/pdf/covid/ strategy/New_additional_Advisory_23062020_2.pdf. [Last accessed on 2020 Jul 04].

4. Centers for Disease Control, Interim Guidelines for COVID-19 Antibody Testing in Clinical and Public Health Settings. Available from: https:// www.cdc.gov/coronavirus/2019-ncov/lab/resources/antibody-testsguidelines.html. [Last accessed on $2020 \mathrm{Jul} 04]$.

5. Singhal T, Shah S, Naik R, et al. Prevalence of COVID-19 antibodies in healthcare workers at the peak of the pandemic in Mumbai, India: a preliminary study. Indian J Med Microbiol 2020;38(3-4):461-463. DOI: 10.4103/ijmm.IJMM_20_308.

6. Murhekar MV, Bhatnagar T, Selvaraju S, et al., Jeromie Wesley Vivian Thangaraj SARS-CoV-2 antibody seroprevalence in India, AugustSeptember, 2020: findings from the second nationwide household serosurvey www.thelancet.com/lancetgh Published online January 27, 2021 10.1016/S2214-109X(20)30544-1. 
7. Malani A, Shah D, Kang G, et al. Seroprevalence of SARS-CoV-2 in slums versus non-slums in Mumbai, India. Lancet Glob Health 2021;9(2):e110-e111. DOI: 10.1016/ S2214-109X(20)30467-8.

8. George C, Inbaraj L, Chandrasingh S, et al. High seroprevalence of COVID-19 infection in a large slum in South India; what does it tell us about managing a pandemic and beyond? Epidemiol Infect 2021;149:E39. DOI: 10.1017/S0950268821000273.

9. Babu GR, Sundaresan R, Athreya S, et al. The burden of active infection and anti-SARS-CoV-2 IgG antibodies in the general population: results from a statewide survey in Karnataka, India. medRxiv 2020. preprint 10.1101/2020.12.04.20243949;.

10. Selvaraju S, Kumar MS, Thangaraj JWV, et al. Chennai COVID sero-surveillance team. Population-based serosurvey for severe acute respiratory syndrome coronavirus 2 transmission, Chennai,
India. Emerg Infect Dis 2021;27(2):586-589. DOI: 10.3201/eid2702. 203938.

11. Baveja S, Karnik N, Natraj G, et al. Rapid volunteer-based SARS-Cov-2 antibody screening among health care workers of a hospital in Mumbai, India. Indian J Med Sci 2020;72(3):148-154. DOI: 10.25259/ IJMS_234_2020.

12. Kataria S, Phogat R, Sharma $P$, et al. Is Covid-19 seroprevalence different in health care workers as per their risk of exposure? A study from a tertiary care hospital in National Capital Region of India. medRxiv 2021. preprint 10.1101/2021.02.10.21251543this version posted February 12, 2021.

13. Singhal T, Shah S, Naik R, et al. Persistence of SARS-CoV-2 antibodies beyond 6 months in health care workers in Mumbai. Indian J Med Microbiol 2021;39(2):267. DOI: 10.1016/j.ijmmb.2021.02.003. 